\title{
Ising Ferromagnetism of Composite Fermions
}

\author{
K. VÝBornýa ${ }^{a, b}$, O. ČertíK ${ }^{a}$, D. PfannKUChE ${ }^{b}$, \\ D. Wodziński ${ }^{c, d}$, A. WóJs ${ }^{c, d}$ AND J.J. QuinN ${ }^{d}$ \\ ${ }^{a}$ Institute of Physics, ASCR, Cukrovarnická 10, 16253 Prague 6, Czech Republic \\ ${ }^{b}$ Institut für Theoretische Physik, Universität Hamburg \\ Jungiusstrasse 9, 20355 Hamburg, Germany \\ ${ }^{c}$ Institute of Physics, Wrocław University of Technology \\ Wybrzeże Wyspiańskiego 27, 50-370 Wrocław, Poland \\ ${ }^{d}$ Department of Physics, University of Tennessee, Knoxville, TN 37996, USA \\ The quantum Hall ferromagnets at the half-filling of a pair of degenerate \\ electron or composite fermion Landau levels are studied by exact numeri- \\ cal diagonalization. The results obtained using open and closed geometries \\ (rectangular — with periodic boundary conditions and spherical) are com- \\ pared. The ferro- and paramagnetic ground states are identified in finite-size \\ energy spectra, and the pair-correlation functions are used in search of the \\ domain structure at half-polarization.
}

PACS numbers: 73.43.-f, 71.10.Pm, 75.10.Jm

\section{Introduction}

The composite fermion (CF) mapping [1] allows understanding of many phenomena related to the fractional quantum Hall effect in terms of the more transparent integral quantum Hall effect. There are two competing ground states with long-range spin order at the integral filling factor $\nu=2$ : a fully spin-polarized state $(P=1)$ and a spin-singlet $(P=0)$. Hence, it was no surprise that two spin orders were experimentally proven also at $\nu=2 / 5$ and $2 / 3$, both corresponding to the filling factor $\nu^{*}=2$ of the CFs. However, later experimental studies of this ferromagnet-paramagnet transition suggested yet another, half-polarized stable ground state [2].

Using exact diagonalization in terms of electrons and CFs, on a torus and on a sphere, we investigate the above filling factors. The $P=1 / 2$ ground state is a correlated state of CFs with spin degree of freedom. We demonstrate that the correct choice of model $\mathrm{CF}-\mathrm{CF}$ interaction is crucial in the description of this 
state, even on the level of energies [3]. Therefore, calculations with electrons are more reliable. An analysis of the wave functions suggests that the $\nu=2 / 3$ system at $P=1 / 2$ is paramagnetic.

At all three fillings, $\nu=2,2 / 5$, and $2 / 3$, the pair of ground states with $P=0$ and 1 are closely related to those of an Ising ferromagnet. In spite of this, we demonstrate that the excitation spectra of these integral and fractional quantum Hall ferromagnets (QHF) show marked differences. Concentrating on the $P=1 / 2$ states, the integral QHF forms domains of $P=0$ and 1 , with domain-wall energy of $0.042 E_{\mathrm{C}}$ (with $E_{\mathrm{C}}=e^{2} / 4 \pi \varepsilon \ell_{0}$ being a convenient Coulomb energy scale) per magnetic length $\ell_{0}=\sqrt{\hbar / e B}$. This behavior, distinct from the paramagnetic ordering of the fractional QHF, stems from different $e-e$ and $\mathrm{CF}-\mathrm{CF}$ interactions. The $\nu=2 / 3 \mathrm{QHF}$ is then an example of a system where the exact form of the $\mathrm{CF}-\mathrm{CF}$ interaction is essential, with experimentally observable consequences.

The essential feature of QHFs is $[4,5]$ that a "unique" ground state exists in a pair of degenerate Landau levels (LLs) with opposite spins ( $\uparrow$ and $\downarrow$ ) holding $n=e B / h$ electrons per unit area, which is exactly half the number of allowed single-particle states. The quotes at the word "unique" mean that this ground state is unique up to a simultaneous rotation of spins of all electrons. These rotations form a subgroup of $S U(2)$. Depending on which two LLs are involved, this can be either $Z_{2}$ (e.g., for $0 \downarrow$ and $1 \uparrow$ ) or $S U(2)$ (e.g., for $0 \downarrow$ and $0 \uparrow$ ), denoting the LLs by their orbital index and spin. While spin of the the ground state can be only up or down in the former case (as in Ising ferromagnets), the latter case allows for any spin direction provided all spins are parallel (as in Heisenberg ferromagnets). This long-range spin order, suggesting ferromagnetism terminology, is induced by the $e-e$ interaction, which thus ensures that out of all $n ! / 2 !(n-2)$ ! possible single-electron configurations only the "unique" ground state is selected. Finally, we remark that under the two "active" LLs there may or may not be one or more completely filled LLs, which are usually considered inert. This assumption is reasonable if the energy separation between the "active" and "inert" LLs is much larger than the characteristic interaction energy $E_{\mathrm{C}}$ which gives rise to ferromagnetism. For example, an Ising QHF occurs at $\nu=\varrho /(e B / h)=2$ if the Zeeman splitting is adjusted to equal the cyclotron energy (with $\varrho=N / A$ being electron concentration).

The tool of our study is the exact diagonalization scheme with electrons [6]. The two active LLs in a 2D system are modeled by a rectangle ( $a$ by $b$ ) with periodic boundary conditions. The number of allowed single-electron states with each spin is equal to the number of flux quanta $\Gamma$ that penetrate the rectangle; $\Gamma \phi_{0}=A B$, where $\phi_{0}=h / e$ is the flux quantum and $A=a b$ is the system area. The filling factor is defined as $\nu=N / \Gamma$. On the other hand, for a given flux number $\Gamma$, the area is fixed as $A=2 \pi \ell_{0}^{2} \Gamma$. In order to gain a better control over the finite size effects in this model we were checking our results also with the spherical geometry and compared systems of different sizes whenever possible. 


\section{Composite fermion interaction}

For two particles confined to a pair of LLs, interaction is determined by Haldane pseudopotential defined as pair interaction energy $V$ vs. relative angular momentum $\mathcal{R}$. In the lowest $\mathrm{LL}, V_{00}(\mathcal{R})$ is rather featureless, but the inter-LL interaction $V_{01}(\mathcal{R})$ already shows short-range oscillations due to a node in the charge-density profile of the excited electron. This effect is only enhanced for CFs, with a complicated short-range behavior of $V_{01}^{*}(\mathcal{R})$ and $V_{11}^{*}(\mathcal{R})$, different from the corresponding $e-e$ pseudopotentials [7].

\section{Quantum Hall ferromagnets}

Not only the ground state, but the complete spectrum of an Ising ferromagnet should be invariant under the $S_{z}$ sign reversal (the $Z_{2}$ symmetry). For $\nu=2$ this is indeed the case, see Fig. 1a. On the other hand, the total spin is no longer a good quantum number as a consequence of the truncation of the Hilbert space to the active (crossing) LLs only. Physically, this corresponds to the high $B$ limit when the interaction-induced LLl mixing to the fully occupied LL (0个) becomes negligible.
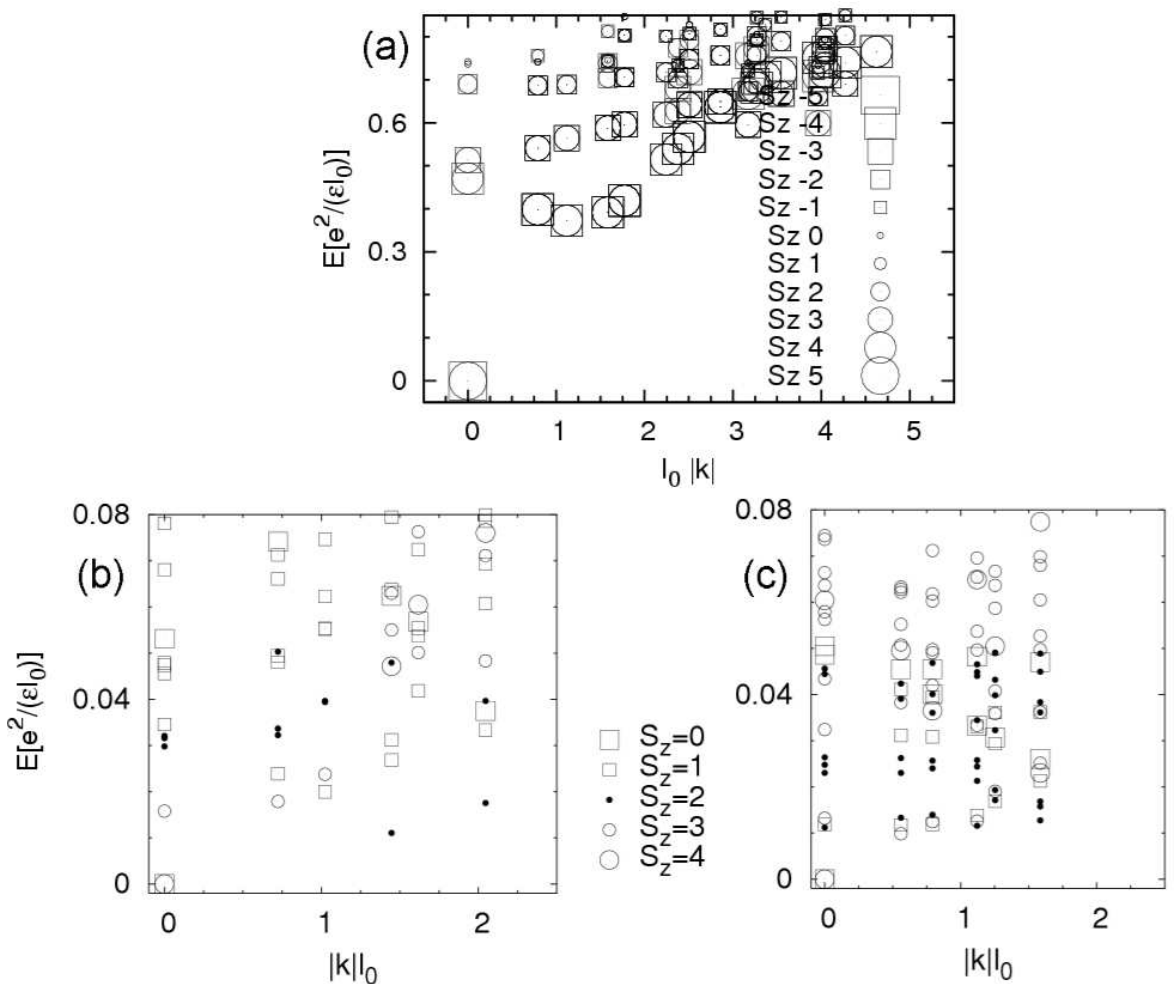

Fig. 1. The excitation spectra of the integral QHF at $\nu=2(\mathrm{a})$, and fractional QHFs at $\nu=2 / 3(\mathrm{~b})$, and $\nu=2 / 5$ (c). 
In contrast to Heisenberg ferromagnets, the ferromagnetic ground state at $\nu=2$ has a gap even for a vanishing Zeeman energy. The lowest excited states contain a single spin flip, and they form a dispersion branch $E(k)$ with a minimum of excitation energy $0.35 E_{\mathrm{C}}$ at a wave number $k \approx 1.4 \ell_{0}^{-1}$.

As shown in Fig. 1 b, the picture at $\nu=2 / 3$ and $2 / 5$ is quite different. The gap above the $Z_{2}$-symmetric ground state is still present, and it is approximately an order of magnitude smaller than in the integral case, as can be expected for the reduced charge of the involved Laughlin quasi-particles. However, no well-resolved dispersion branches can be identified in the excitation spectrum. More importantly, it is not clear whether the lowest excitation has one or more spin flips. Particular attention should be paid to the absence of the $Z_{2}$ symmetry in the active CF LLs. (In the electronic states this symmetry makes two $z$-components of the total spin, $S_{z}$ and $N / 2-S_{z}$, equivalent to each other.) At this point, differences between $\nu=2 / 3$ and $2 / 5$ should be highlighted. In the latter system, a group of low energy states appears in which $Z_{2}$-related pairs of similar energy can be identified. Such a structure is not observed at $\nu=2 / 3$.

These findings are not surprising given the existent coupling between the "active" CF LLs and the completely filled $0 \uparrow$ which is not excluded in the electronic calculation. The spin polarization of these low-lying states breaks the $Z_{2}$ symmetry and this effect cannot be eliminated (e.g., in the high $B$ limit) because the interaction which effectively couples different CF LLs is one and the same as the interaction which creates the CF LLs themselves. This is a substantial difference between the integral and fractional QHFs.

It is remarkable that states with $P=1 / 2$ lie quite low in the excitation spectra at both $\nu=2 / 3$ and $2 / 5$ (in Fig. 1b, c these are the states with $\left.S_{z}=2\right)$. Among these, the lowest two states at $\nu=2 / 3$ have been studied using the spin-resolved density-density correlation functions shown in Fig. $2 \mathrm{~d}$, e, $\mathrm{f}$ for one of the states for $N=12$. They indicate that their internal structure is very similar, in particular if the elementary cell is slightly elongated (aspect ratio $a: b<2$ ). Two pronounced maxima along the short side of the elementary cell are observed which hint at paramagnetic spin ordering. As shown in Fig. 3b, the variations of energy of these states under varying aspect ratio are neither strong nor monotonous. Hence, the elongation probably affects the correlations in the states but it is unlikely to change their structure fundamentally. At this point, a comparison of systems with different sizes would be very useful to decide whether these states are generic to infinite $2 \mathrm{D}$ systems, or if they occur solely due to the system's finite size. Unfortunately, with present computational capacity, the next larger system $(N=16)$ is inaccessible for either direct exact diagonalization. Alternatively, the density matrix renormalization group (DMRG) method [8] would probably have to be used. Noteworthy, the spectrum of the next smaller system $(N=8)$ also reveals two states with similar properties as described above, supporting the idea of paramagnetic 

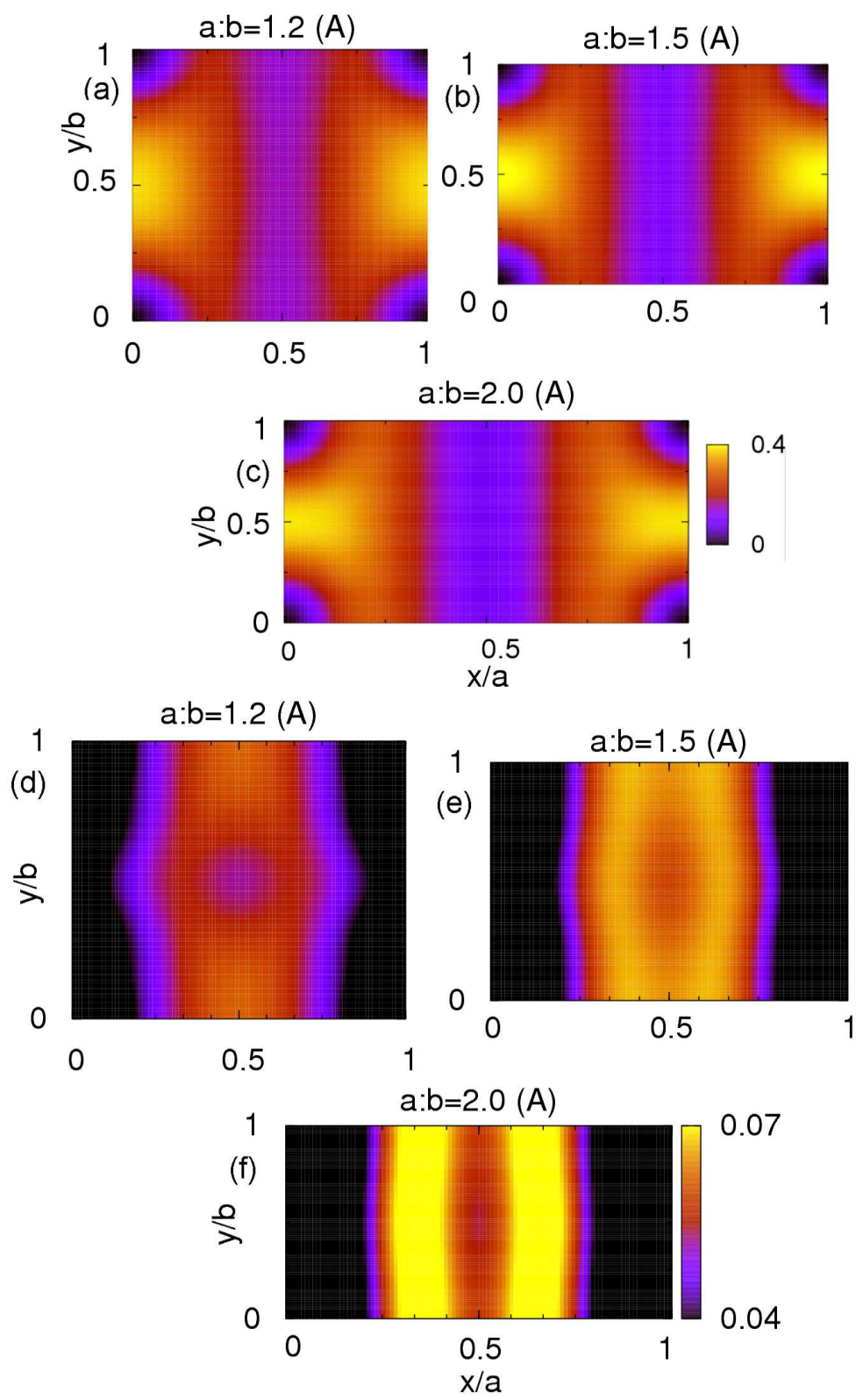

Fig. 2. Spin-resolved pair correlation functions $g_{\downarrow \downarrow}(\boldsymbol{r})=\left\langle\delta\left(\boldsymbol{r}_{12}-\boldsymbol{r}\right) \delta_{\sigma_{1}}^{\downarrow} \delta_{\sigma_{2}}^{\downarrow}\right\rangle$ in the lowest $1 / 2$-polarized states for different aspect ratios. (a-c) $\nu=2 ;(\mathrm{d}-\mathrm{f}) \nu=2 / 3$.

ordering. Nevertheless, these conclusions should be taken with appropriate reservation as they are based on comparison of only two different and rather small systems. 

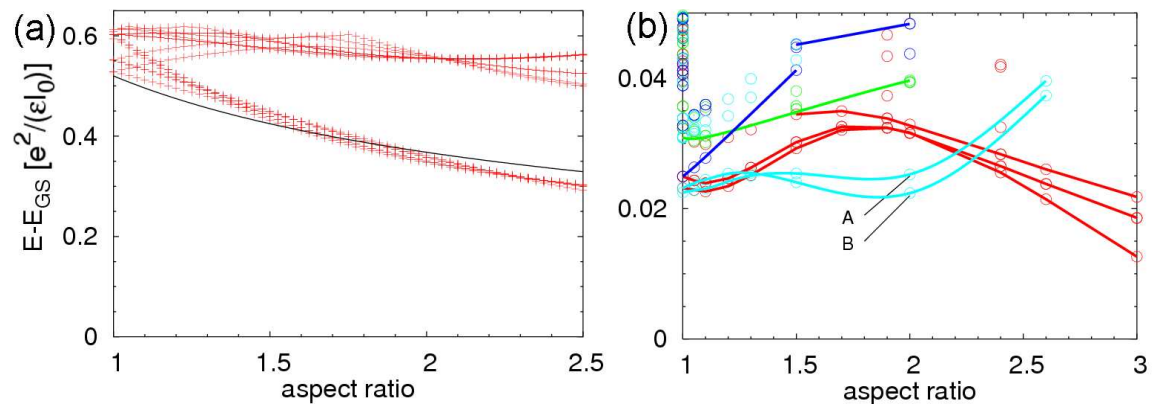

Fig. 3. Energy of the lowest half-polarized states under varying aspect ratio $a: b$ of the elementary cell. (a) $\nu=2, N=6$ electrons, inert LL neglected; solid line is a fit $0.52 / \sqrt{\alpha}, \sqrt{\alpha} \propto b$. (b) $\nu=2 / 3, N=12$ electrons, inert CF LL included. In both cases, energy of ferromagnetic ground states is set to zero.

The half-polarized states at $\nu=2$ give a quite different picture. Already a slight elongation $(a / b \approx 1.2)$ creates a group of states which are clearly separated from the higher excited ones, Fig. 3a. Again, the internal structure of all states in the group is nearly identical. However, in contrast to $\nu=2 / 3$, the group counts not two but $N$ states, i.e. the number of states in the low-energy group grows with the system size. The correlation functions in Fig. 2a, b, c clearly identify two equally sized domains of $P=0$ and $P=1$, separated by two domain walls parallel to the shorter side of the elementary cell. Within the group, the states differ by the location of the domain wall [9]. The largest part of the observed energy variation is simply the domain wall creation energy, which was estimated to $0.042 E_{\mathrm{C}}$ per magnetic length $\ell_{0}$ and drawn with a solid line in Fig. 3a. On top of this variation, slight changes induced by the deformation of the domain "bulk" are still observable.

\section{Conclusion}

There is a correspondence between quantum Hall systems at filling factors $\nu=2$ and $\nu=2 / 3$ or $2 / 5$. As far as the ground state is considered, they pose examples of Ising QHFs. This relation is apparent in the CF picture, in which both fractional states correspond to the integral CF filling $\nu^{*}=2$.

The lowest excitation at $\nu=2$ seems to be a gaped spin wave with $E_{\mathrm{g}}=$ $0.35 E_{\mathrm{C}}$. This may be a likely scenario also at $\nu=2 / 5$ and at $\nu=2 / 3$ - with $E_{\mathrm{g}} \approx 0.01 E_{\mathrm{C}}$ - even though the non-negligible and irreducible mixing between the inert and active CF LLs makes the excitation spectra somewhat unclear and also not quite identical at $\nu=2 / 5$ and $2 / 3$. Ultimately, it cannot be excluded that states other than single spin waves will become lower in energy in larger systems. A good candidate for the lowest excitation of $\nu=2 / 3$ and $2 / 5$ may be half-polarized states. However, extrapolation of the energies to the thermodynamical limit did not yield a conclusive answer. 
The correlation functions at $\nu=2 / 3$ suggest unidirectional paramagnetic spin order in the active LLs. This is very different from half-polarized states at $\nu=2$, which form spatially separated domains with $P=0$ and 1 .

As far as numerical models are considered, a conclusive answer to the question of the lowest excitation at $\nu=2 / 3$ or $2 / 5$ can only be given by further studies in larger systems. Exact diagonalization in terms of CFs could be advantageous, however a precise understanding of the interaction between CFs at different partial occupations of LLs is an imperative.

\section{Acknowledgment}

K.V., O.Č. acknowledge support from grants AV0Z10100521 of the Academy of Sciences of the Czech Republic and LC510 of the Czech Republic Center for Fundamental Research. D.W., A.W., J.J.Q. acknowledge support by DOE Basic Energy Science and grant 1PO3B03330 of the Ministry of Science and Higher Education (Poland).

\section{References}

[1] J.K. Jain, Phys. Rev. Lett. 63, 199 (1989); Science 266, 1199 (1994).

[2] I.V. Kukushkin, K. Klitzing, K. Eberl, Phys. Rev. Lett. 82, 3665 (1999).

[3] V.M. Apalkov, T. Chakraborty, P. Pietiläinen, K. Niemelä, Phys. Rev. Lett. 86, 1311 (2001).

[4] T. Jungwirth, A.H. MacDonald, Phys. Rev. B 63, 035305 (2000).

[5] K. Výborný, PhD thesis, Universität Hamburg, Hamburg 2005, www.sub.unihamburg.de/opus/volltexte/2005/2553.

[6] T. Chakraborty, P. Pietiläinen, The Quantum Hall Effects, Springer, Berlin 1995.

[7] A. Wójs, D. Wodziński, J.J. Quinn, Phys. Rev. B 71, 245331 (2005).

[8] N. Shibata, D. Yoshioka, Phys. Rev. Lett. 86, 5755 (2001).

[9] E.H. Rezayi, T. Jungwirth, A.H. MacDonald, F.D.M. Haldane, Phys. Rev. B 67, 201305(R) (2003). 